\title{
Biofiltro con cascarilla de arroz y pasto vetiver (Chrysopogon zizanioides) para el tratamiento del efluente de la PTAR del INPEC - Yopal, Casanare, Colombia
}

\section{Biofilter with rice husk and vetiver grass (Chrysopogon Zizanioides) for the treatment of effluent from the WWTP of INPEC - Yopal, Casanare, Colombia}

\author{
Biofiltro com casca de arroz e vetiver (Chrysopogon \\ Zizanioides) para o tratamento de efluente da PTAR \\ INPEC - Yopal, Casanare, Colômbia
}

Sandy Katheryne Higuera Infante

Ingeniera Ambiental, Candidata a especialista en Educación Superior a Distancia, Grupo de Investigación Cazao, Semillero Desarrollo Sostenible, Egresada de la UNAD.

Grupo de Investigación CAZAO. Escuela de Ciencias Agrícolas, Pecuarias y del Medio Ambiente ECAPMA. Universidad Nacional Abierta y a Distancia UNAD. Yopal, Casanare, Colombia.

higuera.sandy@gmail.com

\section{Resumen}

En la investigación se evaluó un biofiltro a escala laboratorio para el tratamiento del efluente de la PTAR del Inpec de Yopal, Casanare, cuya finalidad era lograr un efluente de calidad óptima para reúso del agua en riego agrícola. Se contemplaron tres fases, la primera caracterización y análisis de los parámetros microbiológicos como fisicoquímicos del efluente. La segunda fase diseño y construcción del sistema a escala laboratorio del humedal artificial, se construyó en vidrio, usando cascarilla de arroz, intercalada con grava, se sembró pasto vetiver (Chrysopogon. Zizanioides) y se inundó el sistema para que funcionará con flujo subsuperficial, el tiempo de retención hidráulico TRH fue de 3.4 días. La desinfección con cloro se diseñó a partir de una prueba de demanda de cloro. La tercera fase consistió en la operación del sistema a flujo continuo, el seguimiento se realizó a través de 4 muestreos con frecuencia semanal, en dos de estos muestreos se incluyó medición de $\mathrm{DBO}_{5}$, coliformes totales y fecales y conductividad. Los resultados mostraron que la cascarilla de arroz como sustrato funciona bien, las plantas crecieron permanentemente, ayudando a disminuir la carga orgánica. Como conclusión la cascarilla puede ser un sustituto total o parcial de otros sustratos de biofiltros o humedales de flujo subsuperficial, al ser menos costosa y estar disponible especialmente en la región de la Orinoquía. Sin embargo, su uso debe complementarse con un proceso de reoxigenación del agua, ya que su condición anaerobia es un aspecto negativo para los cuerpos de agua receptores de vertimientos.

Palabras clave: agua residual, humedal artificial, flujo subsuprficial 


\section{Abstract}

The research evaluated a biofilter to scale laboratory for the treatment of the effluent from the WWTP of Inpec from Yopal, Casanare, whose purpose was to achieve an effluent of optimum quality for agricultural irrigation water reuse. Three phases were watched, the first characterization and analysis of the microbiological parameters as physicochemical effluent. The second phase design and construction of the system to scale laboratory of the artificial wetland, was built in glass, using rice husks, interspersed with gravel, was sown grass vetiver (C. zizanioides) and flooded the system so that it will work with subsurface flow, hydraulic retention time TRH was 3.4 days. Disinfection with chlorine was designed from a test of chlorine demand. The third phase consisted in the operation of the continuous flow system, monitoring was carried out through 4 samplings often weekly, in two of these surveys included measurement of BOD5, total and faecal coliforms and conductivity. The results showed that the husk of rice as a substrate works well, the plants grew permanently, helping to reduce organic loads. Conclusion quinine can be a substitute all or part of other substrates to biofilters or subsurface flow wetlands, being less costly and be available especially in the region of the Orinoco. However, its use must be complemented with a process of reoxygenation of water, since its anaerobic condition is a negative aspect to the receiving water bodies of dumping.

Key-words: residual water, artificial wetlands, subsurface flow.

\section{Resumo}

Nesta pesquisa foi avaliado um biofiltro em laboratório para o tratamento do efluente da PTAR do Inpec de Yopal, Casanare, com o objetivo de conseguir uma efluente de ótima qualidade para ser reutilizado na irrigação agrícola. Completaram-se três fases, a primeira fase foi a caracterização e análises dos parâmetros microbiológicos e físico-químicos do efluente. A segunda fase foi desenho e construção do sistema em laboratório de um pantanal artificial, a construção foi feita em vidro, usando casca de arroz, intercalada com grava, foi plantado pasto vetiver (C. zizanioides) e o sistema foi alagado para que funcionara com fluxo subsuperficial, o tempo de retenção hidráulico TRH foi de 3,4 dias. A desinfecção com cloro foi feita a partir de um teste de demanda de cloro. A terceira fase foi a operação do sistema de fluxo continuo, o acompanhamento foi realizado através de 4 amostragens com frequência semanal, foi feita a mensuração de $\mathrm{DBO}_{5}$ em duas das amostragens, assim como coliformes totais e fecais e condutividade. Os resultados mostraram que a casca de arroz como substrato funciona bem e a plantas cresceram continuamente, ajudando a diminuir a carga orgânica. Como conclusão, a casca de arroz pode ser um substituto total ou parcial de outros substratos de biofiltros ou pantanais de fluxo subsuperficial ao ser menos custosa e ser disponibilizada especialmente na região da Orinoquia. No entanto, seu uso deve ser complementário com um processo de re-oxigenação da água devido a que sua condição anaeróbia é um ponto negativo para os corpos de água receptores de vertimientos.

Palavras-chave: água residual, pantanal artificial, fluxo subsuperficial.

\section{Introducción}

La escasez de agua en el mundo ha llevado a buscar cada vez más alternativas para el abastecimiento de este vital líquido además de la conservación del mismo. En ese sentido el reúso de las aguas residuales es una estrategia de ahorro y uso eficiente del agua, fundamental para el modelo de desarrollo sostenible. En muchos lugares se ha aumentado el reúso de las aguas residuales por pequeños o grandes agricultores, debido a la escasez de agua de las fuentes hídricas convencionales. Por tal motivo representa un reto seleccionar tecnologías sostenibles para el tratamiento de las aguas residuales domésticas, creando la necesidad de desarrollar herramientas que faciliten la toma de decisiones para la implementación de estos sistemas, basados en tecnologías naturales de depuración, como humedales artificiales (Espinosa, 2014). A nivel Mundial se han establecido directrices para el reúso de las 
aguas residuales, las cuales han aumentado considerablemente en los últimos años, esto debido a la preocupante escasez de agua. Uno de los aspectos importantes en el reúso de agua son los requisitos mínimos de calidad que deben tener las aguas reutilizadas, con el fin de garantizar que organismos patógenos presentes en éstas no entren en contacto con las personas. (Manga, Logreira, \& Serralt, 2001). Actualmente en Colombia se cuenta con una normatividad que incluye criterios de calidad que debe cumplir el agua residual para reúso, de tal manera que no genere daños ni a la salud humana ni al medio ambiente. Según el IDEAM se prevé que para el año 2016 aproximadamente el $70 \%$ de la población urbana en Colombia tendrá un déficit en el suministro de Agua Potable. Esto produciría efectos económicos, sociales y ambientales que se sumaran a la ya complicada problemática de Colombia. (Manga, Logreira, \& Serralt, 2001). Por estas problemáticas se genera la necesidad de crear tecnologías que permitan el reúso del agua residual en riego. En el departamento de Casanare, Colombia, se está creando una cultura para cumplir la norma de reúso de aguas residuales tratadas, resultantes de las actividades de los diversos sectores productivos. La resolución 1207 de 2014 formulada por el Ministerio de Ambiente y Desarrollo Sostenible determina la utilización de aguas residuales tratadas cumpliendo con los criterios de calidad requeridos para el uso que se vaya a destinar, ya sea para el sector industrial, agrícola o para ornato y mantenimiento de áreas verdes. (Pineda, 2014).

\section{Metodología}

Para la evaluación del sistema de tratamiento de aguas residuales con la tecnología de humedales artificiales a escala laboratorio, se tuvieron en cuenta las siguientes actividades: recopilación de información de la PTAR INPEC, diseño de planta a escala laboratorio, y monitoreo y seguimiento a la calidad del agua.

Zona de Estudio: La investigación se desarrolló en el establecimiento penitenciario y carcelario de Yopal, Casanare, Colombia, ubicado en el km 12 Vía Yopal - Aguazul, actualmente cuenta con un terreno de $440.000 \mathrm{~m}^{2}$. De los cuales $64.661 \mathrm{~m}^{2}$ constituyen la infraestructura del penal y el restante se encuentra destinado para el desarrollo de actividades agropecuarias. En el centro se cuenta con aproximadamente 200 reclusos, la Figura 1 muestra las instalaciones del INPEC.

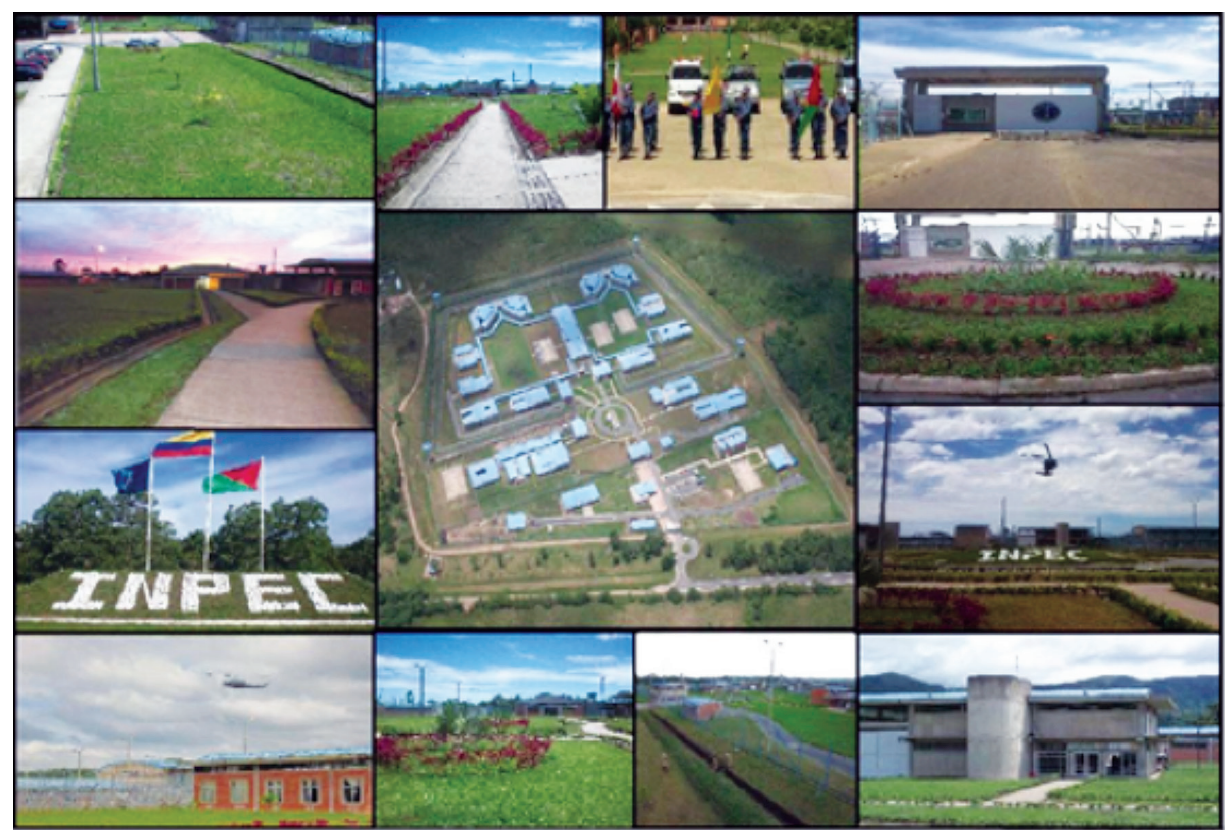

Figura 1. Instalaciones INPEC Yopal

Fuente: http://www.inpec.gov.co 
Descripción de la PTAR existente: La planta de tratamiento de aguas residuales tiene un caudal de entrada de $12 \mathrm{~L} / \mathrm{s}$, es de tipo aerobio, su vertimiento va al caño Guavinas, según los análisis realizados por los laboratorios ANALQUIM la PTAR cumple con la norma para vertimientos. La PTAR del INPEC, se compone de dos reactores biológicos de lodos activados por aireación extendida como tratamiento principal, que permite la remoción de los contaminantes orgánicos presentes en el agua residual que son captados y transportados por el sistema de recolección de aguas residuales dentro del establecimiento. Adicionalmente, el sistema de tratamiento cuenta con estructuras de operación unitaria que permiten la remoción de grasas, aceites, solidos suspendidos, microorganismos patógenos, entre otros, la Figura 2 muestra el diagrama de flujo del sistema de tratamiento existente. (ACUAMEUNIER, 2016).

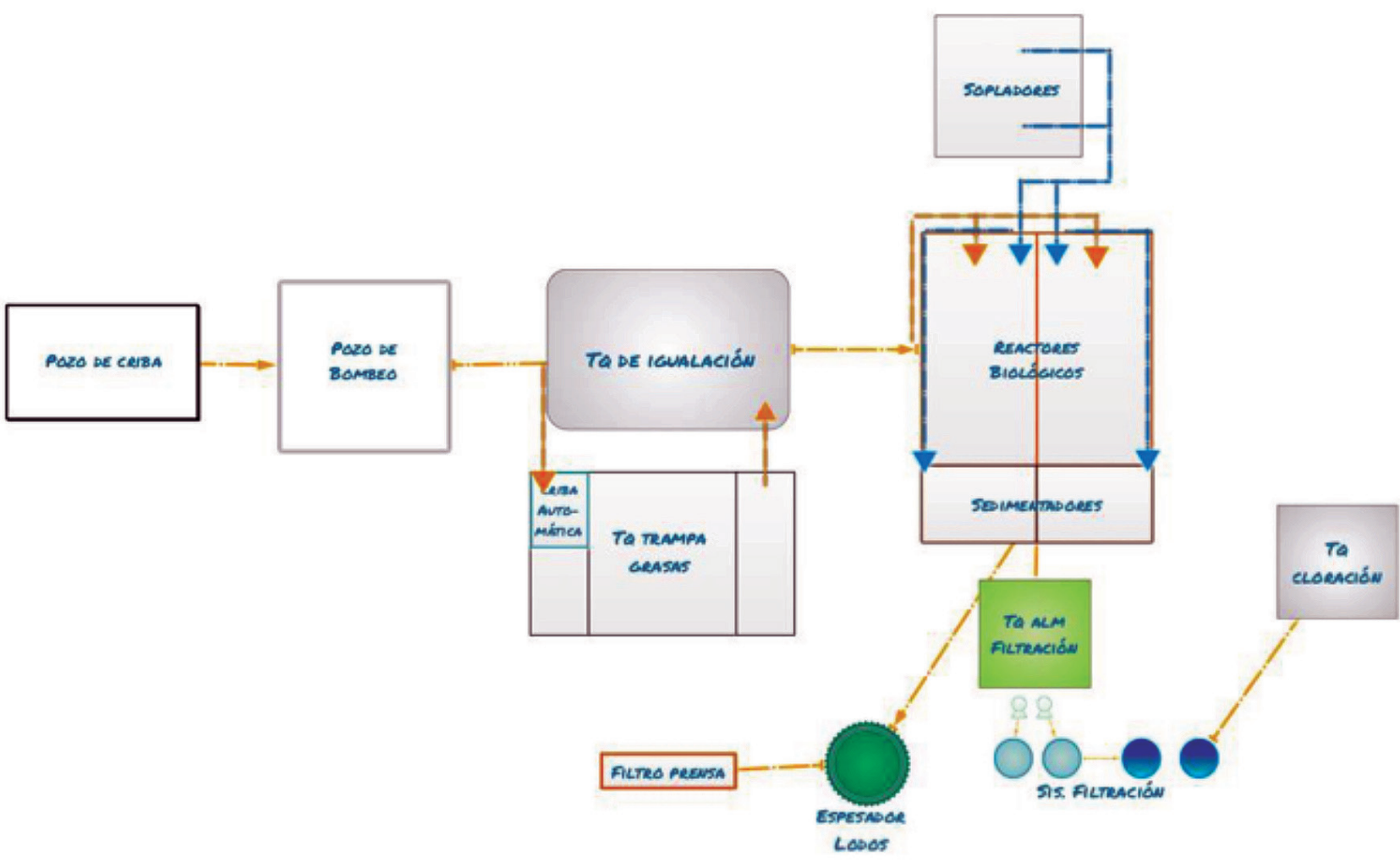

Figura 2 Diagrama de Flujo del sistema de tratamiento

Fuente: ACUAMEUNIER, 2016.

Diseño de prototipo a escala de laboratorio: Para el diseño y construcción del prototipo a escala laboratorio se tuvieron en cuenta dimensiones de acuerdo a la disponibilidad de espacio en el laboratorio del CEAD Yopal, los criterios o recomendaciones para el diseño se tomaron de proyectos similares consultados (Borrero, 1999; Delgadillo, Camacho, Pérez, \& Andrade, 2010: Otálora, 2011), y practicidad en la construcción, en la Tabla 1 se presentan los criterios de diseño del prototipo. 
Tabla 1. Parámetros de diseño del prototipo

\begin{tabular}{l|l|l|l|l|l|l|l}
\hline \multicolumn{7}{|c|}{} & Parámetros de Diseño \\
Sustrato & Plantas & $\begin{array}{c}\text { Tiempo } \\
\text { de Retención } \\
\text { Hidráulico (TRH) }\end{array}$ & $\begin{array}{c}\text { Caudal } \\
\text { Tratado }\end{array}$ & Largo & Ancho & $\begin{array}{c}\text { Profundidad } \\
\text { Total }\end{array}$ & $\begin{array}{c}\text { Profundidad } \\
\text { del Sustrato }\end{array}$ \\
\hline $\begin{array}{l}\text { Grava } \\
\text { y Cascarilla } \\
\text { de Arroz }\end{array}$ & $\begin{array}{l}\text { Pasto } \\
\text { Vetiver }\end{array}$ & 3,4 días & $24 \mathrm{ml} / \mathrm{min}$ & $1,5 \mathrm{~m}$ & $50 \mathrm{~cm}$ & $50 \mathrm{~cm}$ & $0,35 \mathrm{~m}$ \\
\hline
\end{tabular}

Teniendo en cuenta las dimensiones del prototipo se realizó el cálculo del caudal, teniendo en cuenta la porosidad del sustrato (grava $35 \%$ y cascarilla $54 \%$ ), el promedio de las dos es $44,5 \%$, y el TRH se obtuvo realizando aforos del caudal de salida del tanque de almacenamiento entre un rango de 13,51 $\mathrm{ml} / \mathrm{min}$ a $26,3 \mathrm{ml} / \mathrm{min}$, lo que dio como resultado un TRH medio de $3.4 \mathrm{~d}$. Teniendo el promedio del tiempo de retención hidráulica que se trabajó en el humedal se halló el caudal deseado con la ecuación 1.

\section{$\mathrm{Q}=\mathrm{V} / \mathrm{TRH}$}

Primero se determina el volumen útil del sustrato en $\mathrm{m}^{3}$ teniendo en cuenta la porosidad del sustrato y el volumen interno, de acuerdo a ecuación 2.

$$
V=0.2625 \mathrm{~m}^{3 *} 44,5 \%=0,117 \mathrm{~m}^{3}
$$

Una vez obtenido se divide el volumen por el TRH obtenido de los aforos, ecuación 3

$\mathrm{Q}=0,117 \mathrm{~m}^{3} / 3,4$ días $=0.0344 \mathrm{~m}^{3} / \mathrm{d}$

Se realiza una conversión $\mathrm{L} / \mathrm{d}$ y luego a $\mathrm{mL} / \mathrm{min}$ para poder realizar el aforo diario teniendo en cuenta el caudal que se obtuvo, ecuación 4.

$\mathrm{Q}=0.0344 \mathrm{~m}^{3} / \mathrm{d}^{*}=23,8 \mathrm{~mL} / \mathrm{min}$
Con el fin de realizar mejor el aforo manualmente y diariamente, se aproximó el valor del caudal a 24 $\mathrm{mL} / \mathrm{min}$.

Se realizó un seguimiento diario a la operación del sistema del humedal, en el cual se tenía en cuenta revisar el caudal de entrada y salida con el fin de que no se aumentara o disminuyera, se analizaba el crecimiento de las plantas y cambios que tuviera durante su proceso.

Construcción: El sistema se construyó en vidrio de $4 \mathrm{~mm}$ de espesor y dimensiones de: alto: $50 \mathrm{~cm}$, ancho: $50 \mathrm{~cm}$ y largo: $1.5 \mathrm{~m}$.

Alistamiento de sustratos: La cascarilla del arroz se lavó y se desinfecto con agua caliente, la grava de 4-8 inch, únicamente se lavó.

Obtención de las plantas: El pasto vetiver se sembró con un tiempo de germinación de 21 días. El número de plantas sembradas fue de 48 las cuales se sembraron cada $15 \mathrm{~cm}$.

En la Figura 3 se presentan las fases de la construcción del prototipo a escala laboratorio. 


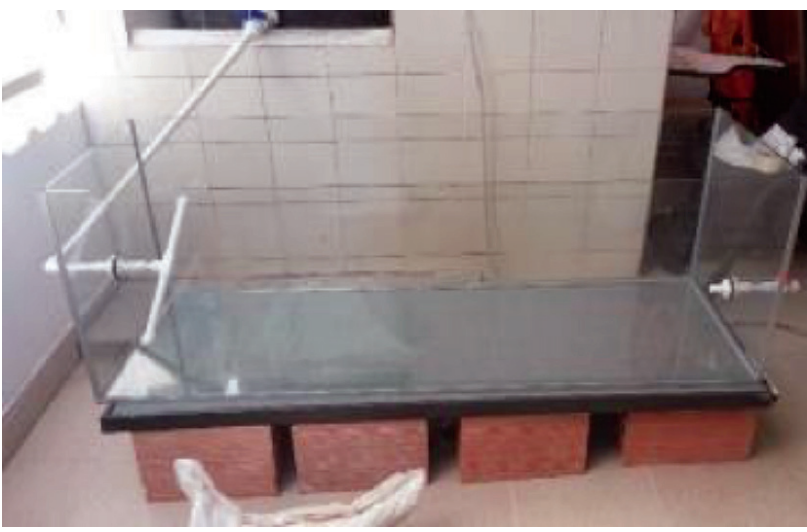

1. Estructura en vidrio y tuberías de entrada y salida de agua

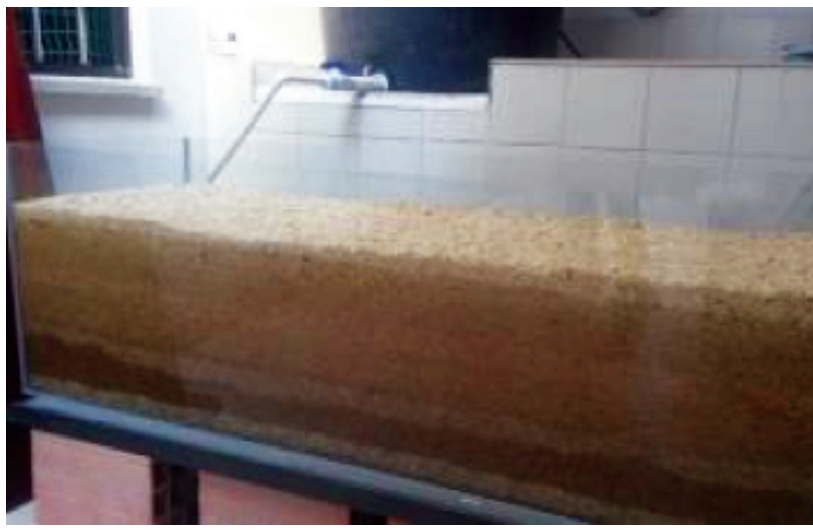

3. Prototipo con sustrato completo a una altura de $35 \mathrm{~cm}$.

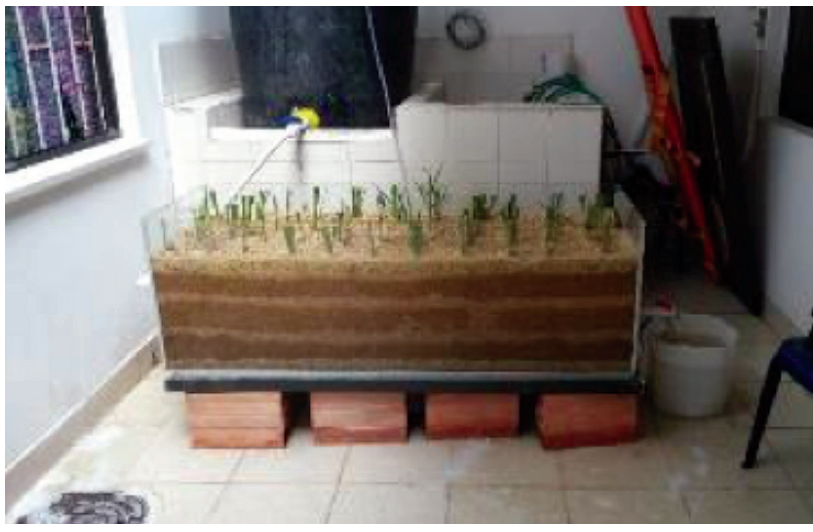

5. Vista del prototipo terminado con plantas, al fondo tanque negro de alimentación

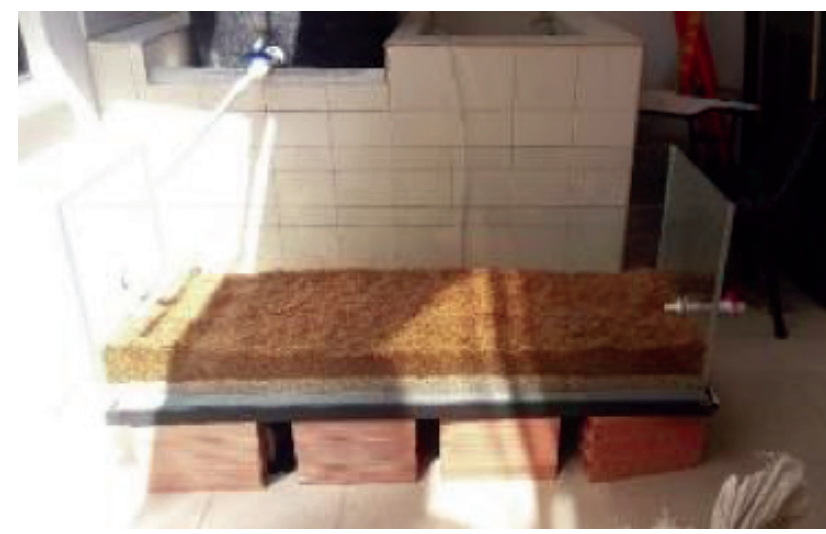

2. Primera capa de sustrato (grava y cascarilla de arroz).

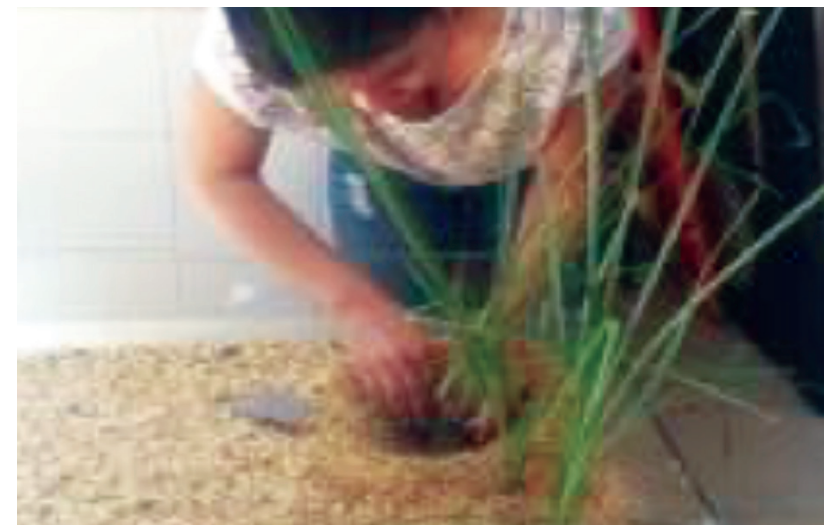

4. Siembra de plantas Vetiver

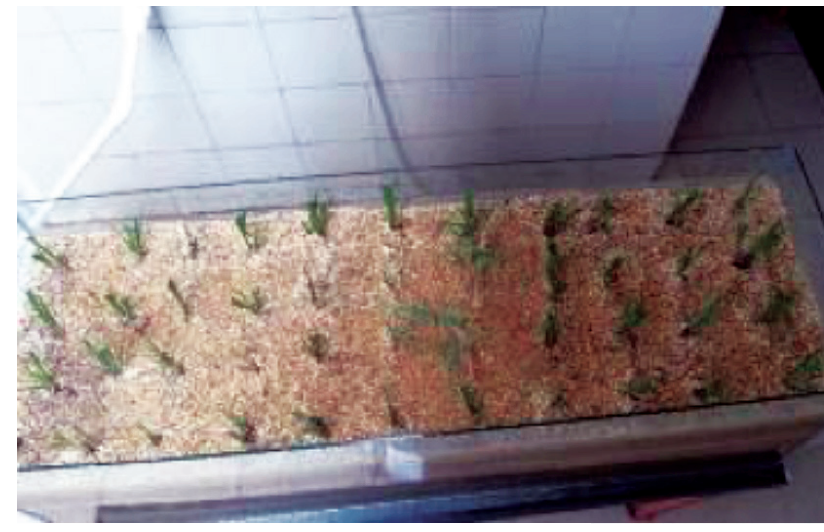

6. Vista del prototipo terminado con plantas

Figura 3. Fotos del prototipo del humedal de flujo subsuperficial

Operación del sistema: Para asegurar el flujo continuo se instalaron dos tanques, un tanque principal de 500L que alimentaba el humedal y otro de $300 \mathrm{~L}$ que alimentaba el tanque principal de manera automática a través del uso de una válvula que accionaba la bomba de alimentación cuando el tanque principal disminuía de nivel. El sistema se operó 1 mes, el monitoreo y seguimiento del sistema se realizó a diario, en el cual se verificó el tiempo de retención hidráulico (TRH) de operación de 3.4 días, 
para eso se midió el caudal de entrada y salida, nivel del agua y flujo del agua, de esta manera se verificaban las condiciones de operación correctas.

Monitoreo y seguimiento a la calidad del agua: Para el monitoreo y seguimiento a la calidad del agua se realizaron muestreos in situ una vez por semana para un total de 4 muestreos y los análisis microbiológicos y otros fisicoquímicos se tomaron dos muestras cada 15 días y se analizaron en los laboratorios AQUALIM en la ciudad de Yopal.

\section{Resultados}

Monitoreo de calidad del agua en el sistema de tratamiento construido: Dentro del cronograma del trabajo estaba realizar muestreos in situ del humedal con el fin de llevar un seguimiento del proceso y los parámetros que se pudieran medir, de estos muestreos se realizaron cuatro los cuales se muestran en las Tablas 2, 3, 4, 5, 6 y 7 donde se puede ver que cumplen con los criterios para reúso del agua en usos agrícolas.

En la Tabla 2 se pueden apreciar resultados obtenidos en el primer muestro, esta indica que hubo incrementos en la dureza y alcalinidad, en cambio se pierde el oxígeno disuelto lo que significa que ha sido consumido para la degradación de la materia orgánica, se mantienen los cloruros, disminuye el $\mathrm{pH}$ a la salida y presencia ausencia de nitritos.

Tabla 2. Primer muestreo in situ

\begin{tabular}{|c|c|c|c|c|c|}
\hline Parámetro & Unidad & Entrada & Salida & Decreto 0631-2015 & Res 1207/2014 \\
\hline Dureza & $\mathrm{mg} / \mathrm{L} \mathrm{CaCO}_{3}$ & 70,5 & 255 & Análisis y Reporte & \\
\hline Sulfitos & $\mathrm{NaSO}_{3}$ & 20 & 19 & & \\
\hline Alcalinidad & $\mathrm{mg} / \mathrm{L} \mathrm{CaCO}_{3}$ & 165 & 225 & & \\
\hline Cloruros & $\mathrm{mg} / \mathrm{l} \mathrm{Cl}$ & 40 & 40 & 250,00 & 300,0 \\
\hline Oxígeno Disuelto & $\mathrm{mg} / \mathrm{L} \mathrm{O}_{2}$ & 1,5 & $\begin{array}{l}\text { No presenta } \\
\text { Oxigeno }\end{array}$ & & \\
\hline $\mathrm{pH}$ & Unidades de $\mathrm{pH}$ & 7,5 & 6,1 & 6,00 a 9,00 & 6,00 a 9,00 \\
\hline Nitritos & $\mathrm{mg} / \mathrm{L} \mathrm{N}-\mathrm{NO} 2$ & 0 & 0 & Análisis y Reporte & \\
\hline
\end{tabular}

En la Tabla 3 en el cual se presentan resultados del segundo muestreo se puede observar que los cloruros se mantienen iguales tanto como en entrada y salida, continua la ausencia del oxígeno disuelto, se encuentra presencia de nitritos, la dureza presenta un significativo aumento a la salida, el pH presenta una disminución a la salida, los sulfitos se mantienen iguales, y la alcalinidad aumenta en la salida. 
Tabla 3. Segundo muestreo - parámetros In-situ

\begin{tabular}{|c|c|c|c|c|c|}
\hline Parámetro & Unidad & Entrada & Salida & Decreto 0631-2015 & Res 1207/2014 \\
\hline Dureza & $\mathrm{mg} / \mathrm{L} \mathrm{CaCO}_{3}$ & 120 & 270 & Análisis y Reporte & \\
\hline Sulfitos & $\mathrm{NaSO}_{3}$ & 20 & 20 & & \\
\hline Alcalinidad & $\mathrm{mg} / \mathrm{L} \mathrm{CaCO}_{3}$ & 150 & 165 & & \\
\hline Cloruros & $\mathrm{mg} / \mathrm{l} \mathrm{Cl}$ & 50 & 50 & 250,00 & 300,0 \\
\hline $\begin{array}{l}\text { Oxígeno } \\
\text { Disuelto }\end{array}$ & $\mathrm{mg} / \mathrm{L} \mathrm{O}_{2}$ & 2 & $\begin{array}{l}\text { No presenta } \\
\text { Oxigeno }\end{array}$ & & \\
\hline $\mathrm{pH}$ & Unidades de $\mathrm{pH}$ & 8 & 7,3 & 6,00 a 9,00 & 6,00 a 9,00 \\
\hline Nitritos & $\mathrm{mg} / \mathrm{L} \mathrm{N}-\mathrm{NO} 2$ & 0,25 & 1.0 & Análisis y Reporte & \\
\hline
\end{tabular}

Para el tercer muestreo como lo muestra la tabla 4 , los cloruros presentan una disminución en la salida, la dureza presenta un aumento bastante alto con respecto a la entrada, los sulfitos aumentan a la salida, el pH disminuye a la salida y los nitritos disminuyeron a la salida.

Tabla 4. Tercer muestreo - parámetros In-situ

\begin{tabular}{|c|c|c|c|c|c|}
\hline Parámetro & Unidad & Entrada & Salida & $\begin{array}{l}\text { Decreto 0631- } \\
\quad 2015\end{array}$ & Res $1207 / 2014$ \\
\hline Dureza & $\mathrm{mg} / \mathrm{L} \mathrm{CaCO}_{3}$ & 105 & 225 & $\begin{array}{l}\text { Análisis y Re- } \\
\text { porte }\end{array}$ & \\
\hline Sulfitos & $\mathrm{NaSO}_{3}$ & 17 & 20 & & \\
\hline Alcalinidad & $\mathrm{mg} / \mathrm{L} \mathrm{CaCO}_{3}$ & 135 & 210 & & \\
\hline Cloruros & $\mathrm{mg} / \mathrm{l} \mathrm{Cl}$ & 80 & 65 & 250,00 & 300,0 \\
\hline $\begin{array}{l}\text { Oxígeno } \\
\text { Disuelto }\end{array}$ & $\mathrm{mg} / \mathrm{L} \mathrm{O}_{2}$ & 0,35 & $\begin{array}{l}\text { No presenta } \\
\text { Oxigeno }\end{array}$ & & \\
\hline $\mathrm{pH}$ & Unidades de $\mathrm{pH}$ & 7,7 & 6,5 & 6,00 a 9,00 & 6,00 a 9,00 \\
\hline Nitritos & $\mathrm{mg} / \mathrm{L} \mathrm{N}-\mathrm{NO} 2$ & 0,25 & 0,05 & $\begin{array}{l}\text { Análisis y Re- } \\
\text { porte }\end{array}$ & \\
\hline
\end{tabular}

La Tabla 5 muestra los primeros análisis realizados en el laboratorio AQUALIM, estos permiten analizar el cumplimiento de los objetivos propuestos en cuanto a la calidad de agua, de acuerdo a los resultados que se presentan en la siguiente tabla se puede observar que el humedal cumple con el $73.25 \%$ de remoción carga orgánica $\left(\mathrm{DBO}_{5}\right)$, su conductividad disminuye y se encuentra dentro de los parámetros permisibles según la resolución 1207/2014, también presenta una disminución de coliformes totales en un $99 \%$ y de E-Coli $99 \%$. 
Tabla 5 .Tercer muestreo - Análisis de laboratorio

\begin{tabular}{|c|c|c|c|c|c|}
\hline \multirow{2}{*}{ Parámetro } & \multicolumn{2}{|c|}{ Resultado } & \multirow{2}{*}{ Unidades } & \multirow{2}{*}{ \% Remoción } & \multirow{2}{*}{$\begin{array}{c}\text { Resolución } \\
1207 / 2014\end{array}$} \\
\hline & Entrada & Salida & & & \\
\hline $\begin{array}{l}\text { Conductividad } \\
\text { Eléctrica }\end{array}$ & 528 & 383 & $\mu$ siemens/cm & $27.5 \%$ & $1.500,00$ \\
\hline $\mathrm{DBO}_{5}$ & 80,0 & 58,6 & $\mathrm{Mg} \mathrm{O}_{2} / \mathrm{L}$ & $26,75 \%$ & \\
\hline $\begin{array}{l}\text { Coliformes } \\
\text { Totales }\end{array}$ & 207500 & 1483 & $\mathrm{NMP} / 100 \mathrm{~mL}$ & $99,28 \%$ & \\
\hline Escherchia Coli & 149700 & 933 & $\mathrm{NMP} / 100 \mathrm{~mL}$ & $99,4 \%$ & $1,0 \star E(+5)$ \\
\hline
\end{tabular}

En el cuarto y último muestreo, cuyos resultados se presenta en la Tabla 6, se observa el resultado del análisis de cloro residual el cual obtuvo un valor de $0,6 \mathrm{mg} / \mathrm{L}$, tomando en cuenta este resultado permite ver que cumple con lo estipulado en la resolución 1207/2014 para reúso del agua residual. De los parámetros medidos in situ se evidencia el incremento de $\mathrm{pH}$ en la salida del humedal, los nitritos presentaron una disminución a la salida del humedal, la alcalinidad presenta un aumento en la salida, los sulfitos aumentaron a la salida, pero la dureza presenta un aumento a la salida.

Tabla 6. Cuarto muestreo - Parámetros in situ

\begin{tabular}{l|l|l|l|l|l}
\hline \multicolumn{1}{|c|}{ Parámetro } & \multicolumn{1}{|c|}{ Unidad } & Salida Humedal & Salida - cloración & $\begin{array}{c}\text { Decreto 0631- } \\
\mathbf{2 0 1 5}\end{array}$ & $\begin{array}{c}\text { Resolución } \\
\mathbf{1 2 0 7 / 2 0 1 4}\end{array}$ \\
\hline Dureza & $\mathrm{mg} / \mathrm{L} \mathrm{CaCO}_{3}$ & 120 & 135 & $\begin{array}{l}\text { Análisis y Re- } \\
\text { porte }\end{array}$ & \\
\hline Sulfitos & $\mathrm{NaSO}_{3}$ & 18 & 20 & & \\
\hline Alcalinidad & $\mathrm{mg} / \mathrm{L} \mathrm{CaCO}$ & 141 & 185 & 6,00 a 9,00 & 6,00 a 9,00 \\
\hline pH & Unidades de pH & 7,3 & 7,1 & Análisis y Re- & \\
\hline Nitritos & $\mathrm{mg} / \mathrm{L} \mathrm{N}-\mathrm{NO} 2$ & 1,0 & 0,15 & & \\
\hline Cloro residual & $\mathrm{Mg} \mathrm{Cl} / \mathrm{L}$ & $<0,1$ & 0,6 & & Menor a 1,0 \\
\hline
\end{tabular}


Los resultados de la Tabla 7 corresponden a análisis realizados en el laboratorio ambiental AQUALIM, es notable el cambio en los resultados obtenidos, ya que aumenta la conductividad eléctrica, la $\mathrm{DBO}_{5}$ en pequeña magnitud, pero si se presenta una remoción completa de coliformes fecales y Escherichia coli.

Tabla 7. Cuarto muestreo - Análisis de laboratorio

\begin{tabular}{|c|c|c|c|c|c|c|}
\hline \multirow[b]{2}{*}{ Parámetro } & \multicolumn{3}{|c|}{ Resultado } & \multirow[b]{2}{*}{ Unidades } & \multirow[b]{2}{*}{ \% Remoción } & \multirow{2}{*}{$\begin{array}{c}\text { Resolución } \\
1207 / 2014\end{array}$} \\
\hline & Entrada & $\begin{array}{c}\text { Salida- } \\
\text { Humedal }\end{array}$ & $\begin{array}{l}\text { Salida - } \\
\text { Cloro }\end{array}$ & & & \\
\hline $\begin{array}{l}\text { Conductividad } \\
\text { Eléctrica }\end{array}$ & 312 & 319 & 4800 & $\mu$ siemens/cm & No hubo remoción & $1.500,00$ \\
\hline DBO5 & 4,2 & 9,9 & $<4,4$ & $\mathrm{Mg} \mathrm{O2/L}$ & No hubo remoción & \\
\hline $\begin{array}{l}\text { Coliformes } \\
\text { Totales }\end{array}$ & 287800 & 5012 & $<10$ & NMP/100 mL & $\begin{array}{l}98.25 \% \text { entre entrada } \\
\text { y salida, pero } \\
100 \% \text { después } \\
\text { de la cloración. }\end{array}$ & \\
\hline Escherichia Coli & 1000 & 20 & $<10$ & NMP/100 mL & $\begin{array}{l}98 \% \text { entre entrada } \\
\text { y salida, pero } \\
100 \% \text { después } \\
\text { de la cloración. }\end{array}$ & $1,0 \star E(+5)$ \\
\hline
\end{tabular}

La diferencia de ese análisis realizado, con respecto al primero puede deberse a la eficiencia del tratamiento en la planta de tratamiento del INPEC, cuando se realizó la primera recolección del agua la planta presentaba una falla en los flotadores y en la última recolección según información del operario la planta estaba trabajando a un $100 \%$, por tal motivo la $\mathrm{DBO}_{5}$ estaba baja.

La Tabla 8 también contiene los resultados del análisis de laboratorio realizado después del sistema de cloración, se observa un incremento de la conductividad eléctrica, este incremento de conductividad se presentó ya que se agregó una cantidad de cloro superior, debido a que la demanda de cloro que se realizó fue de acuerdo al primer muestreo y análisis de laboratorio, el cual presentaba un valor de $\mathrm{DBO}_{5}$ del 58,6 $\mathrm{mg} \mathrm{O}_{2} / \mathrm{L}$, y para el segundo análisis el valor de DBO ya era mucho menor, además de esto se puede deber a presencia de electrolitos en el agua, tal vez dentro del agua se presente algún tipo de jabón, el aumento de la $\mathrm{DBO}_{5}$ puede ser por la cascarilla de arroz como sustrato orgánico, sin embargo presenta una remoción de coliformes fecales y Escherichia Coli.

\section{Análisis de resultados}

Calidad del agua del humedal: Teniendo en cuenta los resultados obtenidos y analizando la eficiencia del humedal a escala piloto, este sistema de tratamiento logró $99 \%$ de eficiencia en la remoción de coliformes fecales y Escherichia Coli, por lo cual estos organismos patógenos cumplen con los criterios de calidad que establece la norma para reuso de aguas residuales, por el contrario, durante el proceso se perdió el oxígeno disuelto esto se debió a la degradación de la materia orgánica, por ende esto desarrolla un medio anaerobio con microorganismos anaerobios y facultativos. Otros parámetros como la $\mathrm{DBO}_{5}$ y la alcalinidad aumentaron durante una fase del tratamiento, una de las posibles causas pudo ser por la cascarilla de arroz como sustrato orgánico ya que este aporta materia orgánica, el comportamiento del $\mathrm{pH}$ se mantuvo constante tanto a la entrada como a la salida del humedal, la conductividad presento una disminución de casi el $28 \%$ a la salida del humedal. 
Calidad del agua con cloración: La calidad del agua residual después del sistema de cloración tuvo cambios significativos en coliformes fecales $y$ Escherichia Coli ya que se removieron en un $100 \%$, por el contrario se presentó un incremento de la conductividad eléctrica, este incremento de conductividad se presentó ya que se agregó una cantidad de cloro superior, debido a que la demanda de cloro que se realizó fue de acuerdo al primer muestreo y a los resultados de los análisis de laboratorio en que la $\mathrm{DBO}_{5}$ fue de $58 \mathrm{mg} / \mathrm{L}$, mientras que el segundo fue $4 \mathrm{mg} / \mathrm{L}$, este tipo de cambios se presentaron por mejoramiento en la eficiencia del sistema de tratamiento que estaba realizando la PTAR del INPEC.

Coeficiente de remoción: Los procesos de remoción de materia orgánica en humedales de flujo subsuperficial son muy efectivos, siempre y cuando se mantengan condiciones óptimas como el tiempo de retención hidráulica, carga orgánica de entrada y profundidad del humedal, este factor "K" es usado para modelar el comportamiento en la remoción de materia orgánica. "K" es la constante de cinética de reacción de primer orden en función de la temperatura, $d-1$, esta se modifica por la temperatura según la ecuación de Arrhenius. (Otálora, 2011). La remoción de $\mathrm{DBO}_{5}$ teniendo en cuenta la cinética de velocidad de reacción "K" fue de $0.091 \mathrm{~d}^{-1}$ lo que indica que, aunque el humedal funcionó muy bien removiendo algunos contaminantes, la constante de remoción de $\mathrm{DBO}_{5}$ es baja en comparación a otros sistemas similares donde esta constante oscila entre 1.1 y $1.35 \mathrm{~d}^{-1}$ (Otálora, 2011), lo cual pudo deberse a una baja carga orgánica en el sistema, también al corto tiempo de operación del sistema y a la variación en la calidad del agua que se trató.

\section{Conclusiones}

Teniendo en cuenta los análisis y parámetros del agua tratada por el humedal, se observa que ésta puede ser reutilizada en el riego de pastos o en cultivos agrícolas y que cumple los requisitos ambientales.
De acuerdo con la eficiencia de remoción de la $\mathrm{DBO}_{5}$ para todo el sistema de un $73,25 \%$ se evidencia el funcionamiento de los humedales artificiales en la remoción de materia orgánica.

El uso de la cascarilla de arroz como sustrato orgánico en el humedal, fue de gran importancia ya que, ésta presenta óxidos metálicos que hacen que se aumente la alcalinidad y disminuye la acidez del agua, por ende, se disminuyen las grasas dentro del tratamiento.

Este tipo de tecnologías demuestran que una vez instalados y operados adecuadamente los humedales artificiales, tienen un funcionamiento adecuado para la remoción de contaminantes en el agua.

En cuanto a las plantas sembradas en el humedal, se puede decir que se adaptaron al sustrato usado, lo que demuestra la viabilidad de usar la cascarilla de arroz como sustrato en humedales artificiales.

Según los resultados obtenidos, se evidencia que el humedal puede alcanzar mayores porcentajes de remoción de material orgánico y organismos patógenos en niveles óptimos de carga orgánica y flujo continuo de agua, esto mejoraría la calidad del agua para reúso agrícola.

\section{Literatura citada}

1. Acuameunier, S. (2016). Manual de operación y mantenimiento planta de tratamiento de agua residual E.P.C. Yopal, Casanare.

2. Analquim, A. F. (2015). Informe de Monitoreo y Caracterización de Agua Residual Domestica. Bogotá D.C.

3. Borrero, J. A. (1999). Depuración de las Aguas Residuales Municipales Con Humedales Artificiales. Recuperado de http://www.ecohabitar.org/wp-content/ uploads/2015/09/humedales-artificiales.pdf

4. Delgadillo, O., Camacho, A., Pérez, L. \& Andrade, M. (2010). Depuración de aguas residuales por medio de humedales artificiales. Recuperado de: http://www. infoandina.org/sites/default/files/publication/files/depuracion_de_aguas_residuales_por_medio_de_humedales_artificiales.pdf 
5. Espinosa, C. E. (2014). Factibilidad del diseño de un humedal de flujo subsuperficial para el tratamiento de aguas residuales municipales de 30.000 habitantes. Escuela Colombiana de Ingeniería - Julio Garavito Recuperado de: http://repositorio.escuelaing.edu. co/bitstream/001/114/1/FACTIBILIDAD\%20DEL\%20 DISENO\%20DE\%20UN\%20HUMEDAL\%20DE\%20 FLUJO\%20SUBSUPERFICIAL\%20PARA\%20 $30000 \% 20 \mathrm{HABITANTES}$.pdf

6. Manga, J., Logreira, N. \& Serralt, J. (2001). Reuso de las aguas residuales. Un recurso hídrico. Ingeniería \& Desarrollo. Universidad del Norte. Recuperado de: http://ciruelo.uninorte.edu.co/pdf/ingenieria_desarroIlo/9/reuso_de_aguas_residuales.pdf
7. Otálora, A. P. (2011). Evaluación del sistema de tratamiento de aguas residuales domésticas mediante humedales artificiales de alta tasa en la locación petrolera de caño Gandúl. Universidad Nacional de Colombia Recuperado de: file://C:/Users/Katy/Downloads/292544.2011.pdf

8. Pineda, C. (2014). Reuso de las Aguas Residuales: Un Beneficio Economico, Social y Ambiental. B. Radio, Entrevistador. 